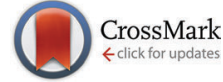

Cite this: Phys. Chem. Chem. Phys., 2016, 18, 19050

Received 11th April 2016, Accepted 16th June 2016

DOI: $10.1039 / \mathrm{c} 6 \mathrm{cp} 02406 \mathrm{~h}$

www.rsc.org/pccp

\section{Dynamics of internally functionalized dendrimers $\uparrow$}

\author{
Jonas Grimm ${ }^{a}$ and Maxim Dolgushev ${ }^{\star a b}$
}

\begin{abstract}
The internally functionalized dendrimers are novel polymers that differ from conventional dendrimers by having additional functional units which do not branch out further. We investigate the dynamics of these structures with the inclusion of local semiflexibility and analyze their eigenmodes. The functionalized units clearly manifest themselves leading to a group of eigenvalues which are not present for homogeneous dendrimers. This part of the spectrum reveals itself in the local relaxation, leading to a corresponding process in the imaginary part of the complex dielectric susceptibility
\end{abstract}

\section{Introduction}

Dendrimers are hyperbranched polymers with a very high symmetry. Due to their perfectly branched structure, dendrimers are promising materials in the fields of chemistry, biology, and medicine, see e.g., ref. 1-3. While the conventional dendrimers were synthesized several decades ago, ${ }^{4-7}$ recently new types of dendrimers have been created, see ref. 8 for review. Among them the internally functionalized dendrimers are of special interest. ${ }^{9-14}$ Their structure differs from homogeneous dendrimers: the internally functionalized dendrimers possess additional functional groups at each branching point (apart from the core). These functional units have distinct chemical properties so that they do not branch out further. As it has been highlighted in ref. 10, there are two major pathways to achieve functionalized dendrimers: premodification (i.e., functionalization before the construction of the macromolecule) or postmodification (i.e., functionalization after the construction of the macromolecule). With respect to these strategies one uses orthogonal protecting groups or groups that can be selectively activated. ${ }^{10}$ In particular, as has been recently shown, ${ }^{13,14}$ the use of orthogonal click reactions based on epoxy-amine and thiol-ene chemistry is very efficient for the introduction of functional groups at each dendritic layer. The advantage of these compounds is that the attachment of drugs or imaging agents to the internal groups can lead to good solubility and biocompatibility properties in comparison to conventional dendrimers. ${ }^{15}$ Moreover, internally functionalized dendrimers can be used as multichromophoric light-harvesting systems, in which the internal functionalization

\footnotetext{
${ }^{a}$ Institute of Physics, University of Freiburg, Hermann-Herder-Str.3, D-79104 Freiburg, Germany. E-mail: dolgushev@physik.uni-freiburg.de

${ }^{b}$ Institut Charles Sadron, Université de Strasbourg \& CNRS, 23 rue du Loess, 67034 Strasbourg Cedex, France

$\dagger$ Electronic supplementary information (ESI) available. See DOI: 10.1039/c6cp02406h
}

helps to establish complex energy gradients. ${ }^{11}$ Furthermore, the layers with different polarities allow the internally functionalized dendrimers to operate as unimolecular catalytic nanosytems. ${ }^{9}$

In this paper we investigate the dynamics of internally functionalized dendrimers. For their description we use the model of semiflexible treelike polymers (STPs) which includes semiflexibility by restrictions on the bonds' orientation. ${ }^{16}$ As has been shown previously for dendritic structures, such orientational restrictions are an important parameter for the characterization of dendrimers. ${ }^{17-21}$ In particular, for the local dynamics, which can be investigated by means of the dielectric or NMR relaxation, ${ }^{22-25}$ the orientational correlations cannot be disregarded. ${ }^{26-28}$ However, the STP framework leads to a higher computational effort stemming from the next-nearest neighboring interactions in the dynamical matrix. Nevertheless, due to the high symmetry of dendrimers, one can block-diagonalize the dynamical matrices very effectively as it has been shown for regular dendrimers. ${ }^{29}$ But, in contrast to regular semiflexible dendrimers ${ }^{29}$ for which the set of eigenmodes of ref. 30 can be readily transferred, for internally functionalized dendrimers the set of eigenmodes of ref. 30 is not straightforwardly applicable. The reason for this feature lies in the symmetry breaking due to the functional units, for which the corresponding degrees of freedom have to be consecutively included. These fundamental degrees of freedom for internally functionalized dendrimers lead to an additional group of eigenvalues, which is not present for regular dendrimers. The ensuing relaxation spectrum leaves its fingerprints on the dynamic properties of the macromolecules. Here we focus on the mechanical and dielectric relaxation, and investigate, in particular, the role of the functional beads.

The paper is structured as follows: in Section 2 we recall briefly the STP-mode ${ }^{16}$ and its formulas for dynamic quantities. In Section 3 we apply the STP-model to the internally functionalized dendrimers. Then, we introduce a complete set of eigenmodes which is used for the diagonalization of dynamic 
matrices. (The recursive construction of the resulting reduced matrices is relegated to Appendix A). We close Section 3 by analysis of the dynamics of internally functionalized dendrimers. Finally, Section 4 presents an overview of the most important results.

\section{Theoretical methods}

\subsection{The model}

The dynamics of internally functionalized dendrimers is modeled in the STP framework, ${ }^{16}$ which we recall here briefly.

The STP model describes polymers as treelike structures of $N$ beads that are connected by springs. Moreover, the bond variables (i.e. springs) $\left\{\mathbf{d}_{a}\right\}$ are constrained to have: (i) fixed mean-square lengths, $\left\langle\mathbf{d}_{a} \cdot \mathbf{d}_{a}\right\rangle=l^{2}$; (ii) two adjacent bonds $\mathbf{d}_{a}$ and $\mathbf{d}_{b}$, connected by bead $i$, fulfilling $\left\langle\mathbf{d}_{a} \cdot \mathbf{d}_{b}\right\rangle= \pm l^{2} q_{i}$, where $q_{i}$ denotes the stiffness parameter of bead $i$, the plus sign applies for a head-to-tail orientation of $\mathbf{d}_{a}$ and $\mathbf{d}_{b}$ and the minus sign for all other configurations of $\mathbf{d}_{a}$ and $\mathbf{d}_{b}$; (iii) for two nonadjacent bonds $\mathbf{d}_{a}$ and $\mathbf{d}_{c}$, connected by the path $\left(b_{1}, b_{2}, \ldots\right.$, $\left.b_{k-1}, b_{k}\right)$, one has $\left\langle\mathbf{d}_{a} \cdot \mathbf{d}_{a}\right\rangle=\left\langle\mathbf{d}_{a} \cdot \mathbf{d}_{b_{1}}\right\rangle\left\langle\mathbf{d}_{b_{1}} \cdot \mathbf{d}_{b_{2}}\right\rangle \cdots\left\langle\mathbf{d}_{b_{k}} \cdot \mathbf{d}_{c}\right\rangle l^{-2 k}$.

As has been shown in ref. 16, the above listed restrictions (i)-(iii) on bonds $\left\{\mathbf{d}_{a}\right\}$ lead to the potential energy $V\left(\left\{\mathbf{d}_{a}\right\}\right)$,

$$
V\left(\left\{\mathbf{d}_{a}\right\}\right)=\frac{K}{2} \sum_{a, b} W_{a b} \mathbf{d}_{a} \cdot \mathbf{d}_{b},
$$

where $K$ is the spring constant that is related to temperature $T$ and the mean-squared bond length $l^{2}$ by $K=3 k_{\mathrm{B}} T / l^{2}$. In eqn (1) the matrix $\mathbf{W}=\left(W_{a b}\right)$ is very sparse; its nonzero elements are either diagonal or related to adjacent bonds. It was shown in ref. 16 that the restriction (iii) follows from maximizing entropy under constraints (i) and (ii) and therefore no Lagrange multipliers associated with restriction (iii) are needed, i.e., the corresponding entries of $\mathbf{W}$ are zeros.

We note that the vectors $\left\{\mathbf{r}_{i}\right\}$ labeling the beads' positions are related to the bonds' vectors $\left\{\mathbf{d}_{a}\right\}$ through

$$
\mathbf{d}_{a}=\sum_{i}\left(\mathbf{G}^{\mathrm{T}}\right)_{a i} \mathbf{r}_{i}
$$

where $\mathbf{G}$ is the so-called incidence matrix ${ }^{31}$ and $\mathbf{T}$ denotes the transposition. Each line of $\mathbf{G}^{\mathrm{T}}$ is related to a bond and contains only two nonzero entries $(+1$ and -1$)$ on the places related to the beads connected by the bond. ${ }^{31}$ Transforming eqn (1) to bead variables (using eqn (2)) yields the matrix $\mathbf{A}^{\text {STP }} \equiv \mathbf{G W G}^{\mathrm{T}}$. Given that $\mathbf{W}$ couples only adjacent bonds and that the nonvanishing elements of $\mathbf{G}$ are related only to beads directly connected by a bond, the nonzero elements of $\mathbf{A}^{\text {STP }}$ are either diagonal or related to the nearest neighboring $(\mathrm{NN})$ and to the next-nearest neighboring (NNN) beads, vide infra.

The dynamics of a polymer is described by a set of $3 \mathrm{~N}$ Langevin equations. The equation for the $x$-component of vector $\mathbf{r}_{i}$ labeling the position of the $i$ th bead reads

$$
\tau_{0} \frac{\partial}{\partial t} x_{i}(t)+\sum_{j=1}^{N} A_{i j}^{\mathrm{STP}} x_{j}(t)=\tilde{f}_{i}(t) / K .
$$

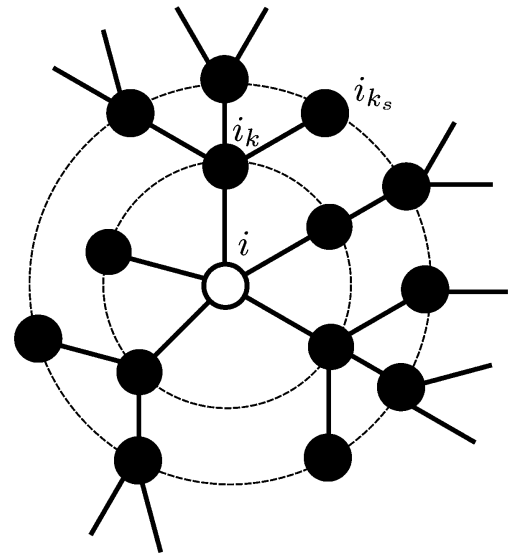

Fig. 1 Nearest (e.g., $i_{k}$ ) and next-nearest (e.g., $i_{k_{s}}$ ) neighbors of a bead $i$ of a treelike network.

Here $\tau_{0}=\zeta / K$, where $\zeta$ is the friction coefficient and $K$ is the spring constant. Moreover, $\tilde{f}_{i}(t)$ is the $x$-component of the stochastic force obeying white noise relations. Furthermore, the structure of a polymer (connectivity and local stiffness) is coded through the matrix $\mathbf{A}^{\text {STP }}=\left(A_{i j}^{\text {STP }}\right)$, whose elements are known in a closed form. ${ }^{16}$ To introduce them, Fig. 1 shows the NN and NNN of a bead $i$ of a treelike structure. The NN beads of $i$ are denoted by $i_{k}$ and the NNN ones by $i_{k_{s}}$. With this notation, the elements of the matrix $\mathbf{A}^{\mathrm{STP}}$ read as follows:

$$
\begin{gathered}
A_{i i}^{\mathrm{STP}}=\frac{f_{i}}{1-\left(f_{i}-1\right) q_{i}}+\sum_{i_{k}} \frac{\left(f_{i_{k}}-1\right) q_{i_{k}}{ }^{2}}{1-\left(f_{i_{k}}-2\right) q_{i_{k}}-\left(f_{i_{k}}-1\right) q_{i_{k}}{ }^{2}}, \\
A_{i_{i_{k}}}^{\mathrm{STP}}=-\frac{1-\left(f_{i}-1\right)\left(f_{i_{k}}-1\right) q_{i} q_{i_{k}}}{\left(1-\left(f_{i}-1\right) q_{i}\right)\left(1-\left(f_{i_{k}}-1\right) q_{i_{k}}\right)}
\end{gathered}
$$

and

$$
A_{i i_{k_{s}}}^{\mathrm{STP}}=\frac{q_{i_{k}}}{1-\left(f_{i_{k}}-2\right) q_{i_{k}}-\left(f_{i_{k}}-1\right) q_{i_{k}}{ }^{2}} .
$$

Here, $f_{i}$ and $f_{i_{k}}$ or $q_{i}$ and $q_{i_{k}}$ denote the functionality or the stiffness of the beads $i$ and $i_{k}$, respectively. All other elements of the matrix $\mathbf{A}^{\text {STP }}$ vanish.

\subsection{Dynamic quantities}

The properties of the matrix $\mathbf{A}^{\mathrm{STP}}$ are fundamental for the dynamics of STP. ${ }^{32,33}$ Using the eigenvalue spectrum of the matrix $\mathbf{A}^{\mathrm{STP}}$ one can compute the complex shear modulus $G^{*}(\omega)=G^{\prime}(\omega)+i G^{\prime \prime}(\omega) \cdot{ }^{34} G^{\prime}(\omega)$ denotes the storage modulus and $G^{\prime \prime}(\omega)$ the loss modulus. Using dimensionless variables one can obtain the reduced moduli ${ }^{33}$

$$
\left[G^{\prime}(\omega)\right]=\frac{G^{\prime}(\omega)}{\nu k_{\mathrm{B}} T}=\frac{1}{N} \sum_{j=2}^{N} \frac{\left(\omega \tau_{0} / 2 \lambda_{j}\right)^{2}}{1+\left(\omega \tau_{0} / 2 \lambda_{j}\right)^{2}}
$$

and

$$
\left[G^{\prime \prime}(\omega)\right]=\frac{G^{\prime \prime}(\omega)}{\nu k_{\mathrm{B}} T}=\frac{1}{N} \sum_{j=2}^{N} \frac{\omega \tau_{0} / 2 \lambda_{j}}{1+\left(\omega \tau_{0} / 2 \lambda_{j}\right)^{2}} .
$$


The sum includes all non-vanishing eigenvalues $\left\{\lambda_{j}\right\}$. The eigenvalue $\lambda_{1}=0$ and its corresponding eigenvector describe the translation of the whole macromolecule.

Using additionally the eigenvectors of the matrix $\mathbf{A}^{\mathrm{STP}}$ one can compute further quantities like the autocorrelation function

$$
M_{1}^{a}(t) \equiv\left\langle\mathbf{d}_{a}(t) \cdot \mathbf{d}_{a}(0)\right\rangle / l^{2}
$$

which turns out to $\operatorname{read}^{27,35}$

$$
M_{1}^{a}(t)=\sum_{j=2}^{N}\left[\left(\mathbf{G}^{\mathrm{T}} \mathbf{Q}\right)_{a j}\right]^{2 \exp \left[-\lambda_{j} t / \tau_{0}\right]} \lambda_{j} .
$$

Here the matrix $\mathbf{Q}$ is constructed from the eigenvectors and the sum runs over all non-vanishing eigenvalues. As we proceed to show in Section 3.2 the eigenvalues $\left\{\lambda_{j}\right\}$ are usually very degenerate for symmetric hyperbranched structures. Denoting the amount of different nonvanishing eigenvalues by $\chi(G)$, eqn (10) can be rewritten as

$$
M_{1}^{a}(t)=\sum_{k=1}^{\chi(G)} C_{k}^{a} \exp \left[-t / \tau_{k}\right]
$$

with $\tau_{k} \equiv \tau_{0} / \lambda_{k}$. The coefficient $C_{k}^{a}$ stands for

$$
C_{k}^{a}=\sum_{n}\left[\left(\mathbf{G}^{\mathrm{T}} \mathbf{Q}\right)_{a n}\right]^{2} / \lambda_{k}
$$

where the sum runs over all eigenvectors belonging to the eigenvalue $\lambda_{k}$. Using this coefficient one can determine through the Fourier-Laplace transform the complex dielectric susceptibility $\varepsilon^{*}(\omega)=\varepsilon^{\prime}(\omega)-\mathrm{i} \varepsilon^{\prime \prime}(\omega)$ for any segment $\mathbf{d}_{a}{ }^{36}$ The real and complex parts read ${ }^{37}$

$$
\varepsilon^{\prime}(\omega)=\sum_{k=1}^{\chi(G)} \frac{C_{k}^{a}}{1+\left(\omega \tau_{k}\right)^{2}}
$$

and

$$
\varepsilon^{\prime \prime}(\omega)=\sum_{k=1}^{\chi(G)} \frac{C_{k}^{a}\left(\omega \tau_{k}\right)}{1+\left(\omega \tau_{k}\right)^{2}},
$$

respectively, where the sums run over the distinct nonvanishing eigenvalues.

\section{Results and discussion}

\subsection{Analysis of $A^{\text {STP }}$ for SFDs}

In this subsection we analyze the matrix $\mathbf{A}^{\mathrm{STP}}$ for semiflexible, internally functionalized dendrimers (SFDs).

First of all, we recall the topology of these structures. The internally functionalized dendrimers differ from homogenous dendrimers by having additional functional beads which do not branch out further. The construction of a functionalized dendrimer is analogous to the homogeneous ones wherein the core bead is connected to $f_{\mathrm{C}}$ beads and all the other beads to $f$ beads. Therefore, the difference $r=f-f_{\mathrm{C}}$ gives the number of functional beads attached to each bead except the core. Thus, setting $f_{\mathrm{C}}=f$ leads to a homogeneous dendrimer of functionality $f_{\mathrm{C}}$. The number of beads of an SFD is hence given by the

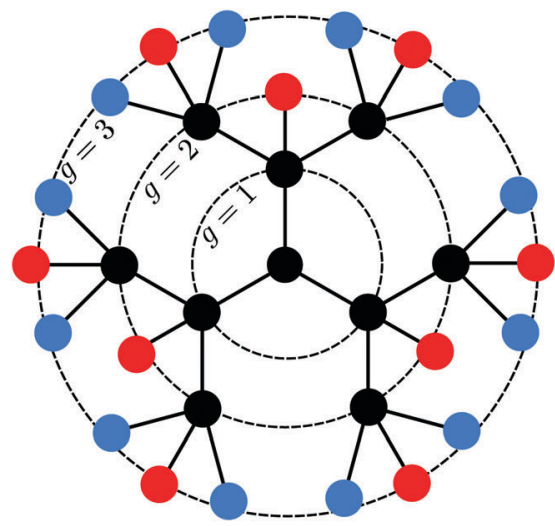

Fig. 2 Schematic drawing of an internally functionalized dendrimer of functionalities $f_{\mathrm{C}}=3$ and $f=4$ and generation $G=3$. The different shells $g=1, g=2$, and $g=3$ are indicated by dashed circles. The functional beads are depicted in red.

number of beads of a homogeneous dendrimer ${ }^{38}$ plus the number of functional beads,

$$
N=\frac{f_{\mathrm{C}}\left(f_{\mathrm{C}}-1\right)^{G}-2}{f_{\mathrm{C}}-2}+r f_{\mathrm{C}} \frac{\left(f_{\mathrm{C}}-1\right)^{G-1}-1}{f_{\mathrm{C}}-2} .
$$

Fig. 2 shows the topology of an internally functionalized dendrimer of functionalities $f_{\mathrm{C}}=3$ and $f=4$ and generation $G=3$.

Based on the SFD structure, the matrix elements of $\mathbf{A}^{\mathrm{STP}}$, which for an SFD we will call in the following by $\mathbf{A}^{\mathrm{SFD}}$, can be readily constructed. We assume a homogeneous situation which means that all internal beads except the core have the stiffness value $q$. The core being different from all other beads has a stiffness value $q_{\mathrm{C}}$. First, we present the diagonal elements $\left\{A_{i i}^{\mathrm{SFD}}\right\}$, then the NN elements $\left\{A_{i i_{k}}^{\mathrm{SFD}}\right\}$, and at last the NNN elements $\left\{A_{i_{i_{k s}}}^{\mathrm{SFD}}\right\}$.

For the diagonal elements one obtains eight different situations (for visualization, see Fig. 3, where the open circles mark the beads corresponding to the discussed matrix element):

(a) If $i$ is a peripheral or functional bead, one has $f_{i}=1$. Moreover, $i$ has exactly one neighbor with functionality $f$ (for $G \geq 2$ ). Using eqn (4), the element $A_{i i}^{\mathrm{SFD}}$, denoted by $\mu_{1}$, equals

$$
\mu_{1}=1+\frac{(f-1) q^{2}}{1-(f-2) q-(f-1) q^{2}} .
$$

(b) For $G=2$, a bead in the first shell has functionality $f$ and possesses $(f-1)$ peripheral neighbors. The core is its inner neighbor with functionality $f_{\mathrm{C}}$. With this, the matrix element reads

$$
\mu_{2}=\frac{f}{1-(f-1) q}+\frac{\left(f_{\mathrm{C}}-1\right) q_{\mathrm{C}}^{2}}{1-\left(f_{\mathrm{C}}-2\right) q_{\mathrm{C}}-\left(f_{\mathrm{C}}-1\right) q_{\mathrm{C}}{ }^{2}} .
$$

(c) For $G>2$, a bead adjacent to a peripheral bead has functionality $f$. Its $(f-1)$ neighbors are peripheral and the 
(a)
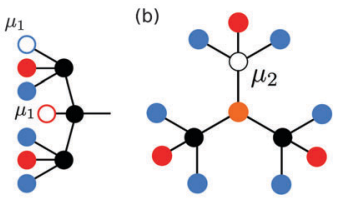

(e)

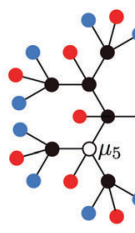

(f)

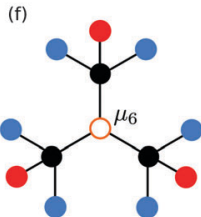

(c)

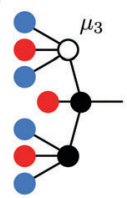

(g)

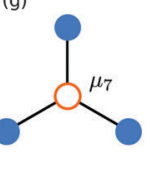

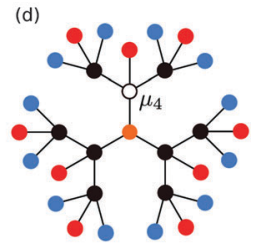

(h)

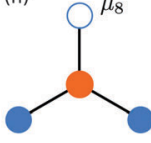

Fig. 3 Schematic drawing represents the diagonal elements of the matrix $\mathbf{A}^{\mathrm{SFD}}$. The core is depicted in orange, the other internal beads in black, the functional beads in red, and the peripheral beads in blue. Open circles mark the beads which are related to the elements $\left\{\mu_{i}\right\}$.

remaining one inner neighbor has functionality $f$. It follows

$$
\mu_{3}=\frac{f}{1-(f-1) q}+\frac{(f-1) q^{2}}{1-(f-2) q-(f-1) q^{2}} .
$$

(d) A bead adjacent to the core has functionality $f$ for $G>2$. Hence, one of its neighbors (namely, the core) has functionality $f_{\mathrm{C}}, r$ neighbors have functionality 1 and $\left(f_{\mathrm{C}}-1\right)$ neighbors have functionality $f$. Therefore, one has

$$
\begin{aligned}
\mu_{4}= & \frac{f}{1-(f-1) q}+\left(f_{\mathrm{C}}-1\right) \frac{(f-1) q^{2}}{1-(f-2) q-(f-1) q^{2}} \\
& +\frac{\left(f_{\mathrm{C}}-1\right) q_{\mathrm{C}}^{2}}{1-\left(f_{\mathrm{C}}-2\right) q_{\mathrm{C}}-\left(f_{\mathrm{C}}-1\right) q_{\mathrm{C}}^{2}} .
\end{aligned}
$$

(e) A bead well inside the dendrimer of $G>2$ has functionality $f$ and possesses $f_{\mathrm{C}}$ neighbors with functionality $f$ and $r$ neighbors with functionality 1 . Therefore, the matrix element reads

$$
\mu_{5}=\frac{f}{1-(f-1) q}+f_{\mathrm{C}} \frac{(f-1) q^{2}}{1-(f-2) q-(f-1) q^{2}} .
$$

(f) The core has functionality $f_{\mathrm{C}}$ and (for $G \geq 2$ ) $f_{\mathrm{C}}$ neighbors with functionality $f$. This leads to

$$
\mu_{6}=\frac{f_{\mathrm{C}}}{1-\left(f_{\mathrm{C}}-1\right) q_{\mathrm{C}}}+f_{\mathrm{C}} \frac{(f-1) q^{2}}{1-(f-2) q-(f-1) q^{2}} .
$$

(g) For $G=1$, the core has $f_{\mathrm{C}}$ neighbors with functionality 1 , so the matrix element reads

$$
\mu_{7}=\frac{f_{\mathrm{C}}}{1-\left(f_{\mathrm{C}}-1\right) q_{\mathrm{C}}} .
$$

(h) For $G=1$, a peripheral bead has one neighbor with functionality $f_{\mathrm{C}}$, therefore, one has

$$
\mu_{8}=1+\frac{\left(f_{\mathrm{C}}-1\right) q_{\mathrm{C}}^{2}}{1-\left(f_{\mathrm{C}}-2\right) q_{\mathrm{C}}-\left(f_{\mathrm{C}}-1\right) q_{\mathrm{C}}^{2}} .
$$

Next, we turn to the NN elements, the corresponding interactions are depicted in Fig. 4 by wavy lines. There are four different elements:(i) If one of the beads is peripheral or functional, the other bead has functionality $f$ (for $G \geq 2$ ).

Eqn (5) then leads to the matrix element, denoted by $\nu_{1}$,

$$
\nu_{1}=-\frac{1}{1-(f-1) q} .
$$

(j) If one of the beads is the core, the other bead has functionality $f$ (if $G \geq 2$ ). Therefore, one gets

$$
\nu_{2}=-\frac{1-\left(f_{\mathrm{C}}-1\right)(f-1) q q_{\mathrm{C}}}{\left(1-\left(f_{\mathrm{C}}-1\right) q_{\mathrm{C}}\right)(1-(f-1) q)} .
$$

(k) If both beads are inside the dendrimer, but none of them is the core, they have functionality $f$, which leads to

$$
\nu_{3}=-\frac{1+(f-1) q}{1-(f-1) q} .
$$

(l) For $G=1$, the element related to the core and to a peripheral bead reads

$$
\nu_{4}=-\frac{1}{1-\left(f_{\mathrm{C}}-1\right) q_{\mathrm{C}}} .
$$

The NNN elements $A_{i i_{k_{s}}}^{\mathrm{SFD}}$ depend only on the bead between the two considered beads, see eqn (6). Therefore, there are two possible distinct elements (the corresponding situations are exemplarily shown in Fig. 4):

(m) If the middle bead is the core, eqn (6) leads to

$$
\rho_{1}=\frac{q_{\mathrm{C}}}{1-\left(f_{\mathrm{C}}-2\right) q_{\mathrm{C}}-\left(f_{\mathrm{C}}-1\right) q_{\mathrm{C}}{ }^{2}} .
$$

(n) If the middle bead is an inner bead with functionality $f$, one has

$$
\rho_{2}=\frac{q}{1-(f-2) q-(f-1) q^{2}}
$$

All other elements of the matrix $\mathbf{A}^{\mathrm{SFD}}$ vanish.

\subsection{Eigenmodes}

In this subsection, we present a complete set of eigenvectors of the matrix $\mathbf{A}^{\mathrm{SFD}}$. The construction of the set is based on the fundamental procedure which was first introduced for flexible, homogeneous dendrimers ${ }^{30}$ (of functionality $f=3$ ) and later generalized for other flexible dendritic structures. ${ }^{38-40}$

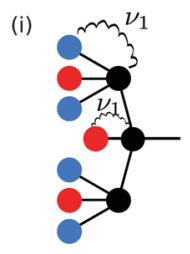

(m)

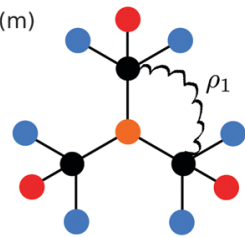

(k)
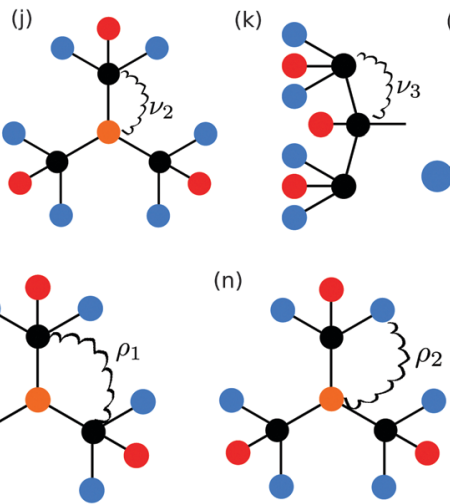

(I)

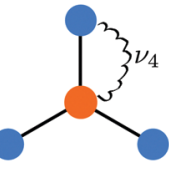

Fig. 4 Schematic drawing of the nonvanishing nondiagonal elements of the matrix $A^{S F D}$, where the corresponding interactions are indicated by wavy lines. The color scheme is as in Fig. 3. 


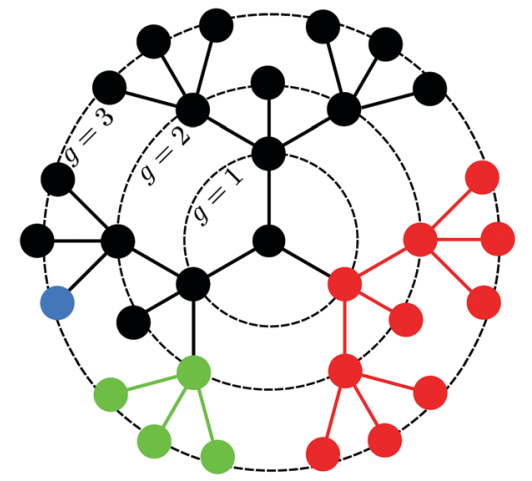

Fig. 5 Functionalized dendrimer of generation $G=3$ with functionalities $f_{\mathrm{C}}=3$ and $f=4$. The color codes different subwedges $\mathscr{Z}^{\left(G_{\mathscr{X}}\right)}: \mathscr{Z}^{(1)}$ (blue), $\mathscr{Z}^{(2)}$ (green), and $\mathscr{Z}^{(3)}($ red).

In ref. 29 it was shown that the same construction of eigenvectors is valid also in the semiflexible case. Here, we extend these methods to examine SFDs of functionalities $f$ and $f_{\mathrm{C}}$ and generation $G$.

Using the symmetry and the SFD structure, the set of eigenvectors can be divided into $(G+1)$ groups. The first group consists of all eigenmodes in which only peripheral beads are moving. The second group consists of the eigenmodes in which the peripheral and their NN are moving. This scheme continues until the group $(G+1)$ is reached in which all the beads including the core are moving, see Fig. 6 and 7 for an illustration.

To be able to describe the eigenmotions of the SFD more accurately, we introduce the notation of the subwedge. A subwedge is a part of the dendrimer which starts with another bead rather than the core. Fig. 5 depicts subwedges $\mathscr{Z}^{\left(G_{\mathscr{X}}\right)}$ of different generations $G_{\mathscr{Z}}$.

As discussed in Section 2.1, the dynamics of SFDs is given by a set of Langevin equations, eqn (3). The corresponding set of homogeneous differential equations reads

$$
\tau_{0} \dot{x}_{l}(t)+\sum_{k=1}^{N} A_{l k}^{\mathrm{SFD}} x_{k}(t)=0 \text { for all } l .
$$

By choosing convenient eigenmodes, the system of equations can be simplified to a few nontrivial equations.

The first group of eigenmodes consists of eigenvectors which describe the movement of two NNN peripheral beads, see Fig. 6. Denoting the two moving beads as $i$ and $j$, the amplitudes $x_{l}$ in eqn (30) read

$$
\begin{gathered}
x_{1} \equiv x_{i}=-x_{j} \\
x_{l}=0 \text { for } i \neq l \neq j .
\end{gathered}
$$

Here, $x_{1}$ labels the single nonvanishing amplitude. Using the respective matrix elements, the single nontrivial equation of motion reads

$$
-\tau_{0} \dot{x}_{1}=\mu_{1} x_{1}-\rho_{2} x_{1}=\left(\mu_{1}-\rho_{2}\right) x_{1} .
$$

All other equations of motion are trivial. For the NN and NNN of the beads $i$ and $j$, the terms of the two moving beads cancel each other because they move with the same amplitude but in the opposite direction. This is sufficient to leave the rest of the dendrimers in rest as well. Therefore, there is one eigenvalue (a)

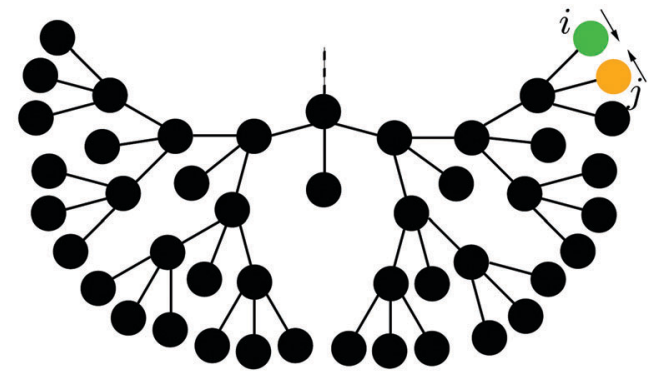

(b)

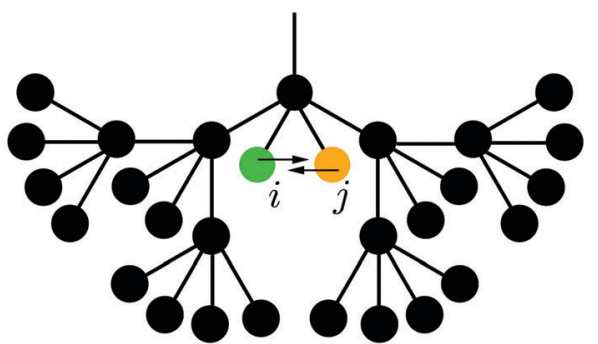

Fig. 6 (a) A sketch of a possible motion related to the first group. (b) A sketch of a possible motion related to the first group involving two functional beads of an SFD of functionalities $f_{\mathrm{C}}=3$ and $f=5$. The moving beads $i$ and $j$ are highlighted by color. See text for details.

belonging to the first group of eigenmodes, denoted by $\lambda^{(1)}$. The described motion involves any two NNN peripheral beads. Each subwedge $\mathscr{Z}^{(2)}$ contains $(f-1)$ peripheral beads which leads to $(f-2)$ different, linear independent eigenvectors. Given that the number of $\mathscr{Z}^{(2)}$ subwedges is equal to $f_{\mathrm{C}}\left(f_{\mathrm{C}}-1\right)^{G-2}$, the eigenvalue $\lambda^{(1)}$ for $r \leq 1$ is $f_{\mathrm{C}}\left(f_{\mathrm{C}}-1\right)^{G-2}(f-2)$-fold degenerate.

In the case of generation $G=1$ one has to use different matrix elements which leads to the equation of motion

$$
-\tau_{0} \dot{x}_{1}=\left(\mu_{8}-\rho_{1}\right) x_{1} .
$$

Furthermore, having $r \geq 2$, the movement of two functional NN beads (see Fig. 6(b)) exactly leads to the same equation of motion as eqn (33) and therefore to the same eigenvalue. Hence, the eigenvalue $\lambda^{(1)}$ is additionally $f_{\mathrm{C}}(r-1) \frac{\left(f_{\mathrm{C}}-1\right)^{G-2}-1}{f_{\mathrm{C}}-2}$-fold degenerate. Altogether, the degeneracy of the eigenvalue connected to the first group is

$$
D_{1}=f_{\mathrm{C}}\left(f_{\mathrm{C}}-1\right)^{G-2}(f-2)+f_{\mathrm{C}}(r-1) \frac{\left(f_{\mathrm{C}}-1\right)^{G-2}-1}{f_{\mathrm{C}}-2} \text {. }
$$

The second group consists of eigenvectors which describe the motion of two subwedges $\mathscr{Z}^{(2)}$ against each other, see Fig. 7(a). Because of symmetry, all topologically equivalent beads of one subwedge move with the same amplitude, the respective beads of the other subwedge with the same amplitude in the opposite direction. There are two eigenvalues belonging to this group, denoted by $\lambda_{1}^{(2)}$ and $\lambda_{2}^{(2)}$. Following an analogous discussion as above, the degeneracy of each of the eigenvalue is

$$
D_{2}=f_{\mathrm{C}}\left(f_{\mathrm{C}}-1\right)^{G-3}\left(f_{\mathrm{C}}-2\right) \text {. }
$$


(a)

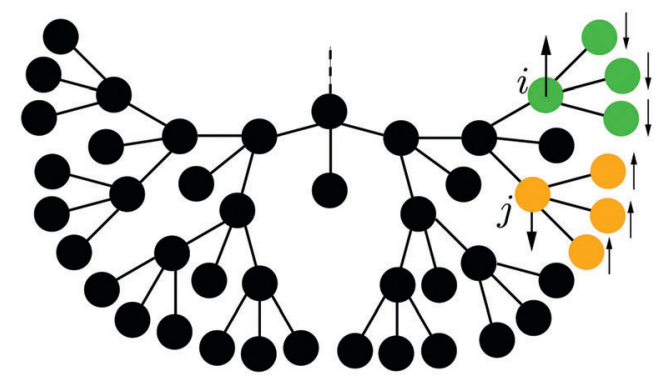

(b)

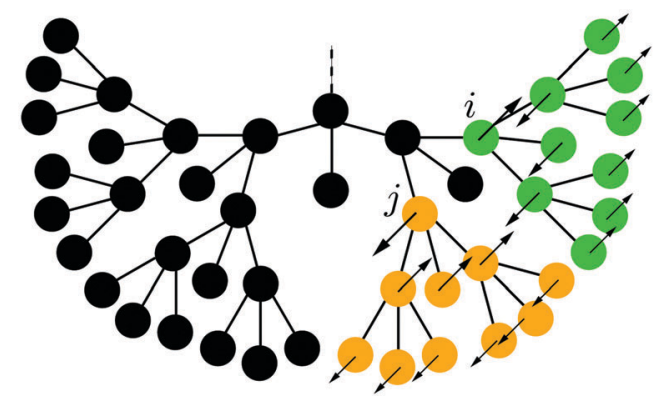

Fig. 7 (a) A sketch of a possible motion related to the second group. (b) A sketch of a possible motion related to the third group. The involved subwedges are colored in green and orange. See text for details.

The third group of eigenmodes describes the motion of two subwedges $\mathscr{Z}^{(3)}$, see Fig. 7(b). Here appears the first structural difference to the homogeneous dendrimer ${ }^{29}$ because the subwedge $\mathscr{Z}^{(3)}$ includes $r$ nonperipheral functional beads. These beads are described by another matrix element than the internal beads in the same shell. Therefore, an extra equation of motion is required. This means that the change in the topology leads to an extra degree of freedom. Hence, there are four eigenvalues connected to the third group of eigenmodes instead of three as it is in the case of homogeneous dendrimers. The respective degeneracy is given by

$$
D_{3}=f_{\mathrm{C}}\left(f_{\mathrm{C}}-1\right)^{G-4}\left(f_{\mathrm{C}}-2\right) .
$$

Now, one additional degree of freedom appears in each iteration from one group to the next one. That means, continuing the procedure leads to two new degrees of freedom for each transition, one for the internal beads of the new involved shell and one for its functionalized beads. Therefore, to the $n$-th group of eigenmodes $(n=2, \ldots, G)$ belongs $2(n-1)$ eigenvalues. The degeneracy is given by

$$
D_{n}=f_{\mathrm{C}}\left(f_{\mathrm{C}}-1\right)^{G-n-1}\left(f_{\mathrm{C}}-2\right)
$$

for $n=2, \ldots,(G-1)$ and by $D_{G}=f_{\mathrm{C}}-1$ for the $G$-th group.

In the group $(G+1)$ all beads including the core are moving. All topologically equivalent beads move with the same amplitude which leads to $(2 G-1)$ degrees of freedom and therefore to $(2 G-1)$ nondegenerate eigenvalues.

To verify that this set of eigenmodes is complete one has to show that their number is equal to the number of beads $N$.
The number of eigenmodes is given by

$$
D_{1}+\sum_{n=2}^{G} 2(n-1) D_{n}+(2 G-1)=N .
$$

Here we used eqn (15) giving the number of beads of an SFD.

\subsection{Reduced matrices}

The knowledge of eigenmodes presented in Section 3.2 allows formulating the respective reduced Langevin equations for each group. The eigenvalues can be then determined by diagonalizing the corresponding reduced matrices.

In the first group of eigenmodes, two peripheral NNN beads move against each other. The Langevin equation is given by

$$
-\tau_{0} \dot{x}_{1}=\mu_{1} x_{1}-\rho_{2} x_{1}=\left(\mu_{1}-\rho_{2}\right) x_{1} .
$$

Hence, the respective eigenvalue reads

$$
\lambda^{(1)}=\left(\mu_{1}-\rho_{2}\right)=\frac{1}{1+q}
$$

for $G \geq 2$. For $G=1, q$ has to be replaced by $q_{\mathrm{C}}$.

The second group leads to the system of equations $(G \geq 3)$

$$
\left\{\begin{array}{l}
-\tau_{0} \dot{x}_{1}=\mu_{1} x_{1}+\nu_{1} x_{2}+(f-2) \rho_{2} x_{1} \\
-\tau_{0} \dot{x}_{2}=\mu_{3} x_{2}+(f-1) \nu_{1} x_{1}-\rho_{2} x_{2} .
\end{array}\right.
$$

For $G=2$, the second equation of set (42) changes to

$$
-\tau_{0} \dot{x}_{2}=\mu_{2} x_{2}+(f-1) \nu_{1} x_{1}-\rho_{1} x_{2} .
$$

The coefficient matrix for these two cases $(G \geq 3$ and $G=2)$ reads

$$
\mathbf{A}_{2}=\left(\begin{array}{cc}
\mu_{1}+(f-2) \rho_{2} & \nu_{1} \\
(f-1) \nu_{1} & \mu_{3,2}-\rho_{2,1}
\end{array}\right) .
$$

Here and in the following, the first index in the last matrix entry has to be used in the case $n<G$ and the second index in the case $n=G$, where $n$ denotes the discussed group of eigenmodes.

For the third group of eigenmodes, one gets the reduced matrix

$\mathbf{A}_{3}$

$$
=\left(\begin{array}{cccc}
\mu_{1}+(f-2) \rho_{2} & \nu_{1} & 0 & \rho_{2} \\
(f-1) \nu_{1} & \mu_{3}+\left(f_{\mathrm{C}}-2\right) \rho_{2} & r \rho_{2} & \nu_{3} \\
0 & \left(f_{\mathrm{C}}-1\right) \rho_{2} & \mu_{1}+(r-1) \rho_{2} & \nu_{1} \\
(f-1)\left(f_{\mathrm{C}}-1\right) \rho_{2} & \left(f_{\mathrm{C}}-1\right) \nu_{3} & r \nu_{1} & \mu_{5,4}-\rho_{2,1}
\end{array}\right) .
$$

Here, different from homogeneous dendrimers, one observes the additional row and column, as discussed in Section 3.2.

By following this procedure one can construct iteratively the matrices up to group $G$. These matrices are relegated to the Appendix.

Next, we discuss the group $(G+1)$. In this group, all beads including the core are mobile. All beads which are topologically equivalent move with the same amplitude. For a dendrimer of generation $G=1$ the system of equations of motion for the 
second group reads

$$
\left\{\begin{array}{l}
-\tau_{0} \dot{x}_{1}=\mu_{8} x_{1}+\nu_{4} x_{2}+\left(f_{\mathrm{C}}-1\right) x_{1} \\
-\tau_{0} \dot{x}_{2}=f_{\mathrm{C}} \nu_{4} x_{1}+\mu_{7} x_{x} .
\end{array}\right.
$$

Therefore, the corresponding reduced matrix is given by

$$
\mathbf{B}_{1}=\left(\begin{array}{cc}
\mu_{8}+\left(f_{\mathrm{C}}-1\right) \rho_{1} & \nu_{4} \\
f_{\mathrm{C}} \nu_{4} & \mu_{7}
\end{array}\right) .
$$

For generations two and three one gets

$$
\mathbf{B}_{2}=\left(\begin{array}{ccc}
\mu_{1}+(f-2) \rho_{2} & \nu_{1} & \rho_{2} \\
(f-1) \nu_{1} & \mu_{2}+\left(f_{\mathrm{C}}-1\right) \rho_{1} & \nu_{2} \\
(f-1) f_{\mathrm{C}} \rho_{2} & f_{\mathrm{C}} \nu_{2} & \mu_{6}
\end{array}\right)
$$

and

$$
\mathbf{B}_{3}=\left(\begin{array}{ccccc}
\mu_{1}+(f-2) \rho_{2} & \nu_{1} & 0 & \rho_{2} & 0 \\
(f-1) \nu_{1} & \mu_{3}+\left(f_{\mathrm{C}}-2\right) \rho_{2} & r \rho_{2} & \nu_{3} & \rho_{2} \\
0 & \left(f_{\mathrm{C}}-1\right) \rho_{2} & \mu_{1}+(r-1) \rho_{2} & \nu_{1} & \rho_{2} \\
(f-1)\left(f_{\mathrm{C}}-1\right) \rho_{2} & \left(f_{\mathrm{C}}-1\right) \nu_{3} & r \nu_{1} & \mu_{4}+\left(f_{\mathrm{C}}-1\right) \rho_{1} & \nu_{2} \\
0 & \left(f_{\mathrm{C}}-1\right) f_{\mathrm{C}} \rho_{2} & f_{\mathrm{C}} r \rho_{2} & f_{\mathrm{C}} \nu_{2} & \mu_{6}
\end{array}\right) .
$$

Based on the spectra of Fig. 8(a) we compute the reduced loss moduli $\left[G^{\prime \prime}(\omega)\right]$ which we show in Fig. 8(b). (We focus on the loss modulus because it typically displays a richer structure than the corresponding storage modulus. ${ }^{33}$ ) As can be inferred from Fig. 8(b), the increasing influence of the eigenvalue $\frac{1}{1+q}$ manifests itself in increasing significance of the left maximum. The growth of the jump after this eigenvalue with a larger amount of functional beads squeezes the region of higher eigenvalues; consequently leading to a shift of the right maximum to higher frequencies.

Now, holding the functionality of the core fixed and varying the amount of functional beads leads to a different functionality of all other beads. Thus, it is also interesting to compare a homogeneous dendrimer of functionality $f=4(r=0)$ and stiffness parameter $q=0.32$ with an SFD of functionalities $f_{\mathrm{C}}=3$ and $f=4$ and stiffness parameters $q_{\mathrm{C}}=0.48$ and $q=0.32$. Therefore, we have a comparable

setup concerning the functionalities and stiffness, while the number of beads of the two dendrimers differs. Fig. 9(a) shows the eigenvalue spectra of the considered dendrimers (and the corresponding SFD spectrum, rescaled with the number of beads). One can see that the spectra look similar after rescaling. The significant difference is the absence of a jump for SFDs in front of the longest plateau as discussed above. There are also minor differences in some of the eigenvalues and in their degeneracy, but they are rather insignificant. Therefore, these two spectra lead to very similar loss moduli as shown in Fig. 9(b). This feature can be traced back to the fact that the periphery of the dendrimers contains a large part of the molecular mass, so that it influences very much the mechanical relaxation, what has been also observed for heterogeneous dendrimers. ${ }^{43}$

Since one can find a homogeneous counterpart to SFDs which lead to similar mechanical relaxation moduli, we are going to look at local properties of such counterparts. The relaxation of single segments is described by the correlation function $M_{1}^{a}(t)$ (see eqn (10)). This quantity requires the knowledge of coefficients involving eigenvectors. Although one can do rigorous calculations of these coefficients by means of projection operator ${ }^{44}$ or reduced description $^{42}$ techniques, here for investigation of the local properties it is enough to perform brute-force computations for an SFD of a smaller generation. Hence in the following we discuss dendrimers of generation $G=5$ and the setup of Fig. 9 .

Fig. 10(a) shows the autocorrelation function $M_{1}^{a}$ for different segments of a homogeneous dendrimer and of an SFD as a function of time. Due to the symmetry, for homogeneous dendrimers the segments belonging to the same shell $g$ have the same dynamics. For SFDs there are two types of segments (functionalized and non-functionalized) in each shell $g>1$, see Fig. 2. In 


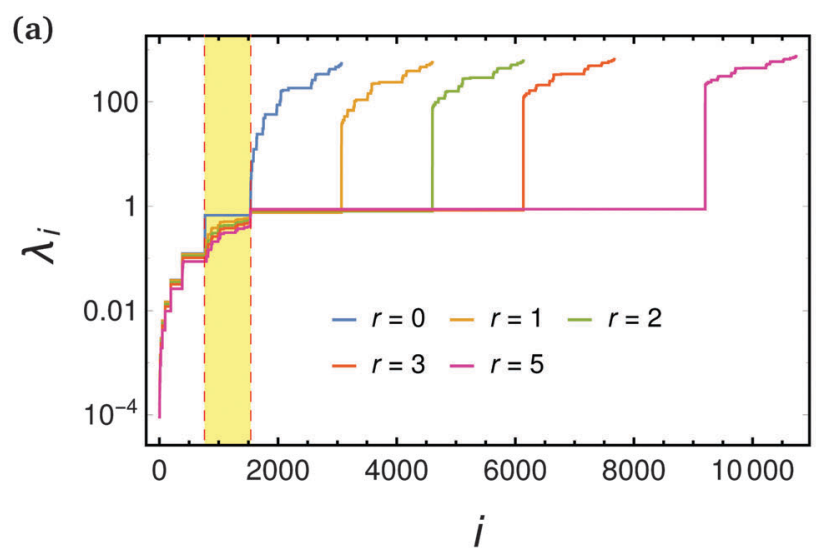

(b)

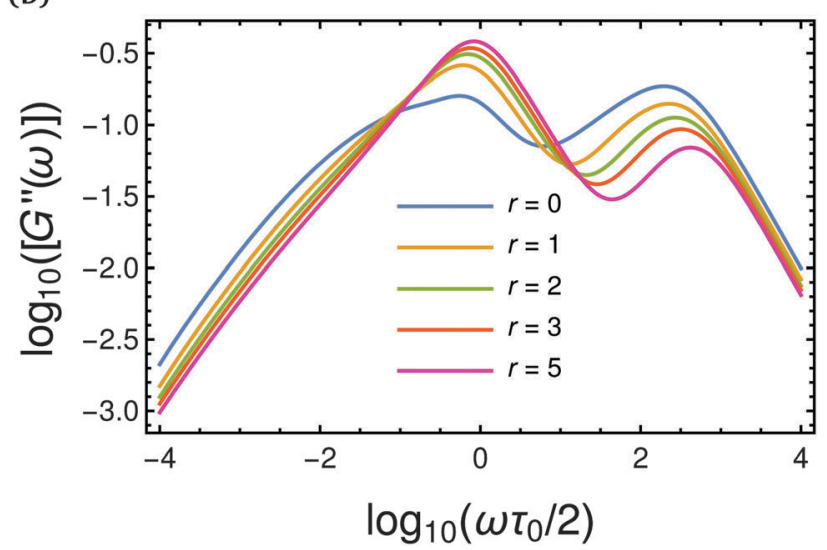

Fig. 8 (a) Eigenvalue spectra and (b) the corresponding reduced loss moduli of an SFD of generation $G=10$ and functionality $f_{C}=3$ with different amounts of functional beads, $r=f-f_{\mathrm{C}}$, see text for details. In (a) the highlighted area indicates the eigenvalues of an SFD $(r \geq 1)$ that originate from the additional degrees of freedom due to the functional beads.

order to have a direct correspondence to the homogeneous dendrimer, we consider for the SFD non-functionalized segments only. One can see that the relaxation of the segments of the homogeneous dendrimer is slower than that of the corresponding ones of the SFD. The difference is smaller for the more peripheral segments. To understand this behaviour we look at the amplitudes $C_{k}^{a}$ of the relaxation decays given by eqn (12) for different relaxation times $\tau_{k}=\tau_{0} / \lambda_{k}$, see Fig. 10(b). As is known, ${ }^{27}$ homogeneous semiflexible dendrimers have one dominating coefficient $C_{k}^{a}$ for each shell. The same behaviour we find for the SFD, as can be observed in Fig. 10(b). However, in the case of SFDs the dominating coefficient has a smaller influence than for the homogeneous dendrimer. Moreover, for SFDs there are some additional amplitudes in the region between $\tau_{0}$ and $10 \tau_{0}$. These amplitudes arise from the eigenvalues in front of the plateau $\frac{1}{1+q}$ in the eigenvalue spectrum, as it was observed in Fig. 8. Furthermore, for each shell $g>1$ the most significant relaxation time of SFDs is smaller than that of the homogeneous dendrimer. These two facts lead to faster decaying correlation functions for higher $g$.

Now, using the amplitude $C_{k}^{a}$ one can compute the real part $\varepsilon^{\prime}(\omega)$ and the imaginary part $\varepsilon^{\prime \prime}(\omega)$ of the complex dielectric susceptibility, see eqn (13) and (14). Here we focus on the $\varepsilon^{\prime \prime}(\omega)$
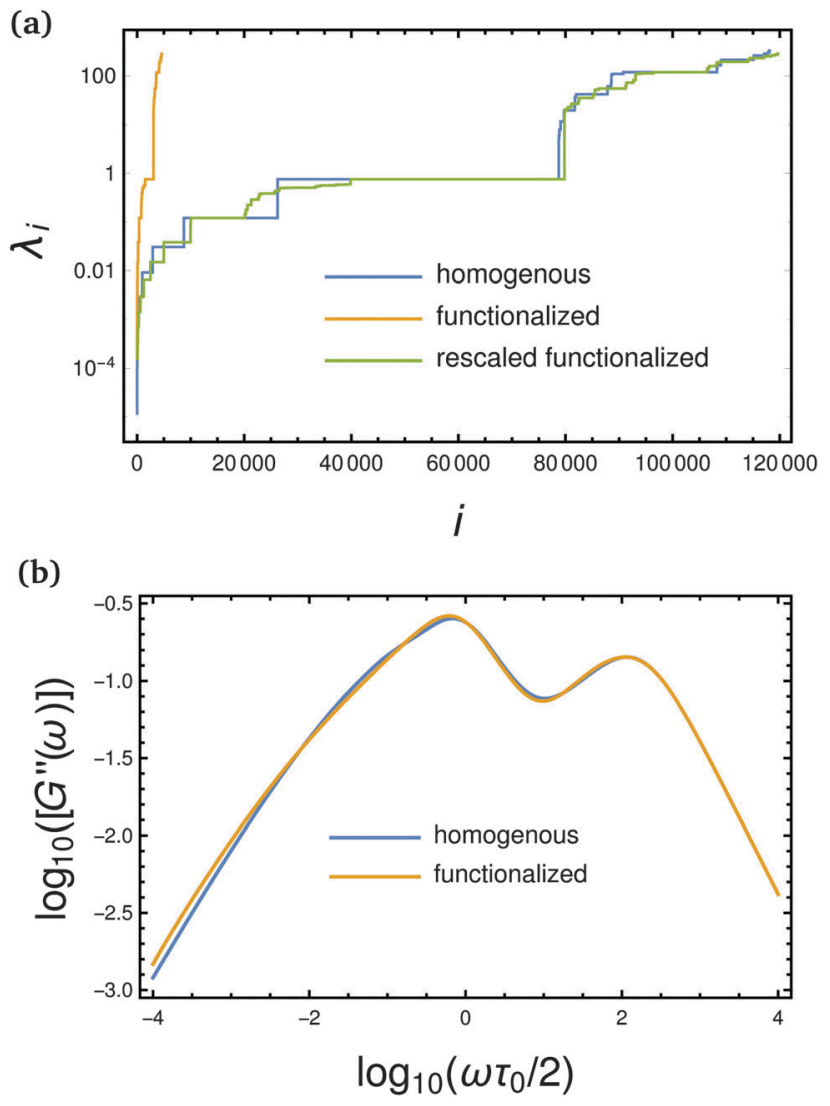

Fig. 9 (a) Eigenvalue spectra of a homogeneous dendrimer $(f=4, q=0.32$ ) and an SFD ( $f_{C}=3, q_{C}=0.48, f=4, q=0.32$ ) of generation $G=10$. The green line represents the rescaled spectrum of the SFD. (b) Reduced loss moduli computed based on the spectra of figure (a). See text for details.

since it displays more emphasized differences. Fig. 11 shows the $\varepsilon^{\prime \prime}(\omega)$ computed based on the amplitudes of Fig. 10(b). As can be inferred from the figure, $\varepsilon^{\prime \prime}(\omega)$ is dominated by two processes. The right maximum originates from the large eigenvalues, i.e. from the relaxation times $\tau_{k}<\tau_{0}$. This maximum is less pronounced since the corresponding amplitudes $C_{k}^{a}$ are small in this region. The left maximum originates mostly from the dominating, very large amplitude. Moreover, for lower $g$ the left maximum appears for the SFD at a bit higher frequencies than for the corresponding shells of the homogeneous dendrimer. This indicates that the viscosity and the gyration radius of the SFD is a bit lower than those of the corresponding homogeneous dendrimer, because the behavior of these characteristics is dominated by lower eigenvalues, see the ESI $\dagger$ for details. Now, for functionalized dendrimers there is an additional shoulder in the intermediate frequency region, which is significantly developed especially for internal shells (i.e. for lower $g$ ). This additional process in the $\varepsilon^{\prime \prime}(\omega)$ arises from the relaxation times between $\tau_{0}$ and $10 \tau_{0}$, see Fig. 10(b). Thus the presence of functional beads is clearly manifested in the local dynamics of the non-functionalized segments on intermediate frequencies. We note that for fully-flexible structures the presence of the additional process in $\varepsilon^{\prime \prime}(\omega)$ is hardly pronounced, i.e., the semiflexibility highlights the presence of functionalized units, see the ESI $\dagger$ for details. 


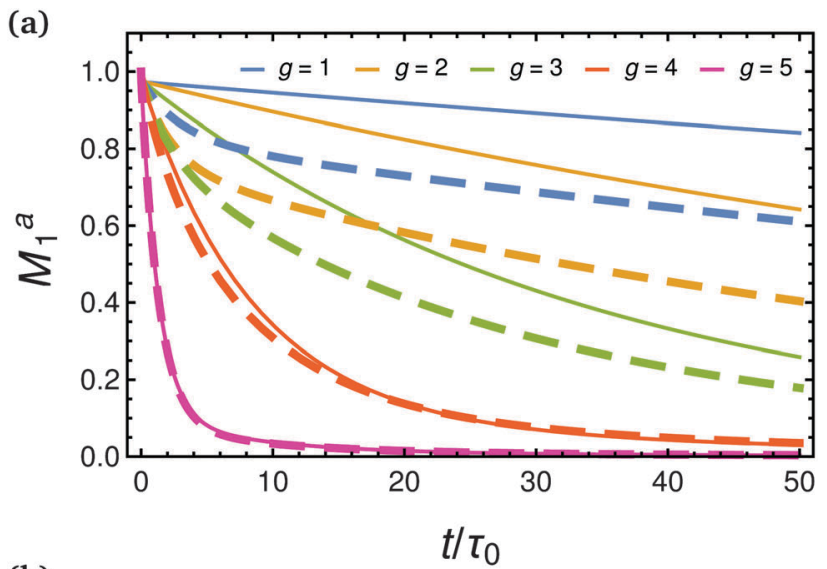

(b)

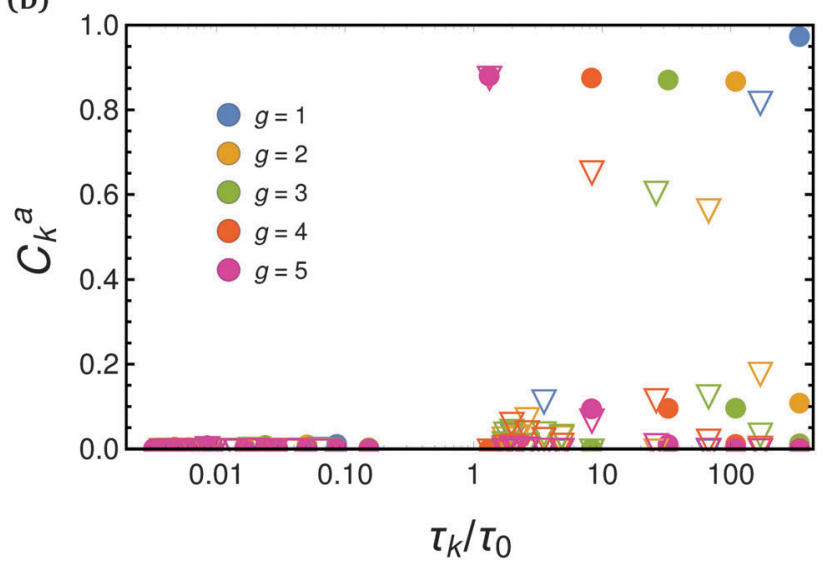

Fig. 10 (a) Autocorrelation function $M_{1}^{a}(t)$ and (b) the corresponding amplitude $C_{k}^{a}$ of the relaxation decays of different segments of a homogeneous $(f=4)$ and a functionalized $\left(f_{C}=3, f=4\right)$ dendrimer of generation $G=5$. In (a) the solid lines are related to segments of the homogeneous dendrimer, while the dashed lines represent the results of the corresponding non-functional segments of the SFD. In (b) the dots indicate the amplitude $C_{k}^{a}$ for the homogeneous dendrimer and the triangles for the SFD. The dendrimers' shells are numbered by $g$ in an ascending order, starting from the core shell, as indicated in Fig. 2.

We close this section by discussing the role of the dendrimers' generation $G$. In Fig. 12 we display the autocorrelation function

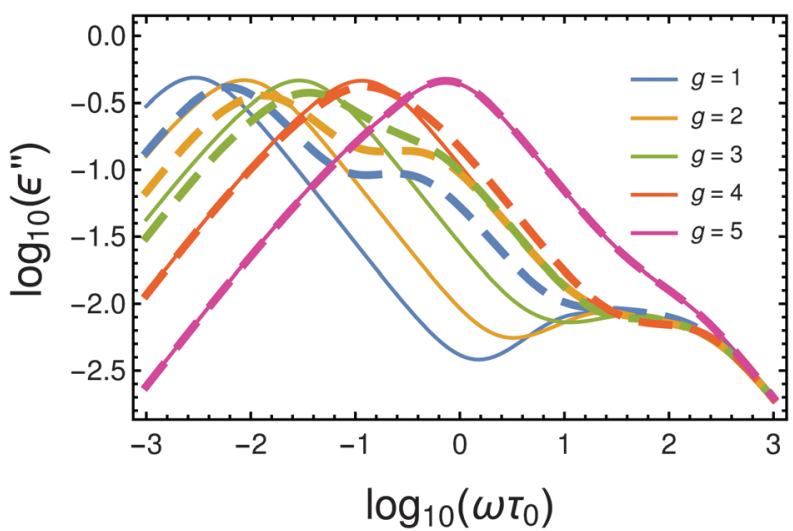

Fig. 11 Imaginary part of the complex dielectric susceptibility corresponding to the structures of Fig. 10. The lines and the color code are the same as in Fig. 10. (a)

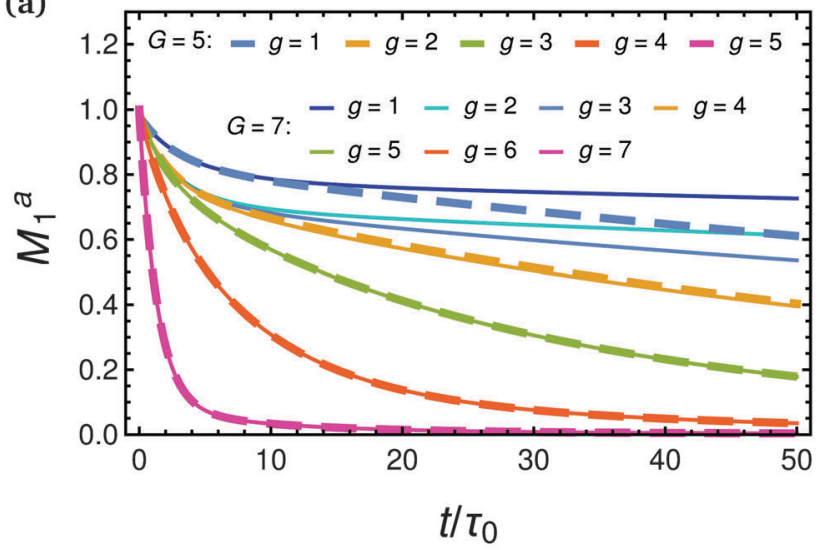

(b)

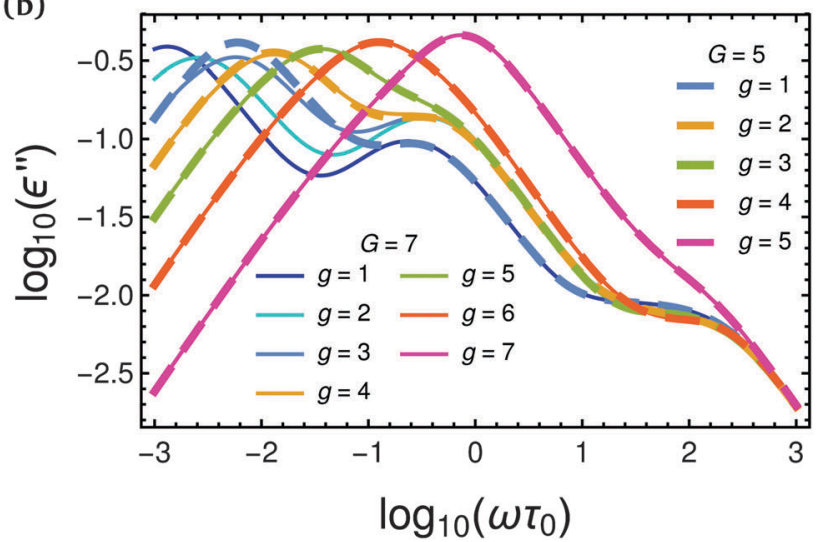

Fig. 12 (a) Autocorrelation function $M_{1}^{a}(t)$ and (b) the corresponding imaginary part of the complex dielectric susceptibility $\varepsilon^{\prime \prime}(\omega)$ for functionalized $\left(f_{C}=3, f=4\right)$ dendrimers of generations $G=5$ (dashed lines) and $G=7$ (solid lines). The dendrimers' shells are numbered by $g$ in an ascending order, starting from the core shell, as indicated in Fig. 2.

$M_{1}^{a}(t)$ and the corresponding imaginary part of the complex dielectric susceptibility $\varepsilon^{\prime \prime}(\omega)$ for different shells $g$ of an SFD of generations $G=5$ and $G=7$. As it was observed for homogeneous dendrimers, ${ }^{27}$ for higher $g$ the curves overlap each other: the corresponding segments feel the size of the branches which originated from them, but not the total size of the dendrimer, i.e., $G$. The behavior of the core segments is somewhat different. Here the difference between the core beads and all other beads is also revealed. In particular, the similarity of neighborhood for core segments of both dendrimers $(G=5$ and $G=7)$ is reflected in the initial decay of $M_{1}^{a}(t)$ or at higher frequencies of $\varepsilon^{\prime \prime}(\omega)$.

\section{Conclusions}

In this work we studied the dynamics of semiflexible, internally functionalized dendrimers (SFDs). Based on their symmetry we have block-diagonalized the dynamical matrix stemming from the STP framework. The ensuing reduced matrices grow linearly with the generation $G$ of the dendrimer, whose molecular mass has an exponential dependence on $G$. The reduced scheme provides not only the computational effort, but also gives a better understanding 
of the SFD's eigenmotions. Starting from generation $G=3$ the SFD eigenmodes become distinct from those of a homogeneous dendrimer. These differences come from the functional beads inside the SFD, resulting in the group of new relaxation times close to the segmental relaxation time. While the mechanical relaxation of SFDs is mostly determined by the peripheral shells, and hence one can find for each SFD a homogeneous counterpart with a similar behavior, the local dynamic properties allow uncovering the presence of functional units. These lead to an additional process (related to the group of new relaxation times close to the segmental relaxation time) in the imaginary part of the complex dielectric susceptibility in the intermediate frequency region.

\section{A Appendix}

Here we present reduced matrices $\mathbf{A}_{n}$ and $\mathbf{B}_{n}$ for $n>3$.

The fourth and fifth groups of eigenmodes lead to the matrices

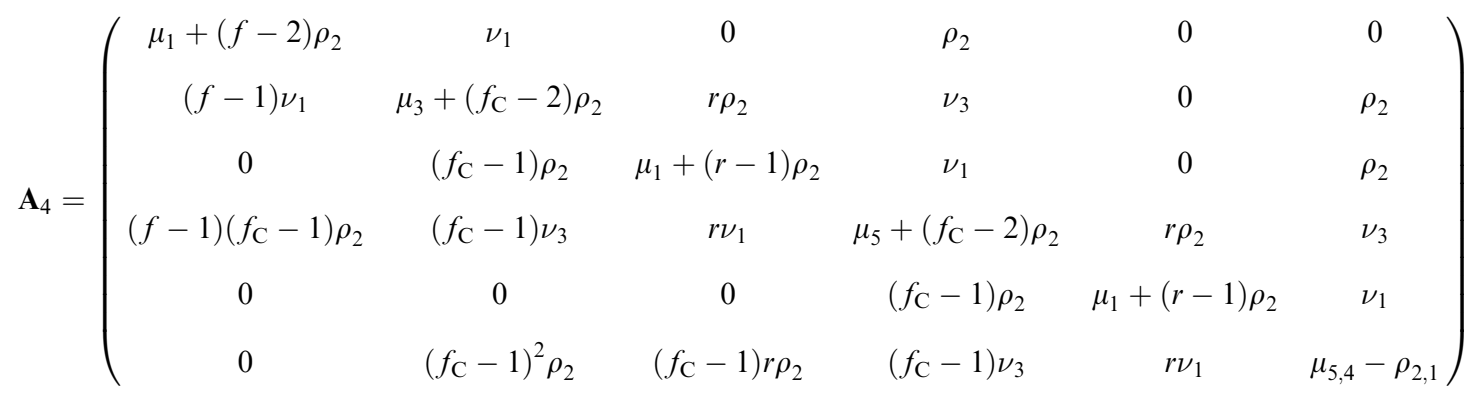

and

$\mathbf{A}_{5}$

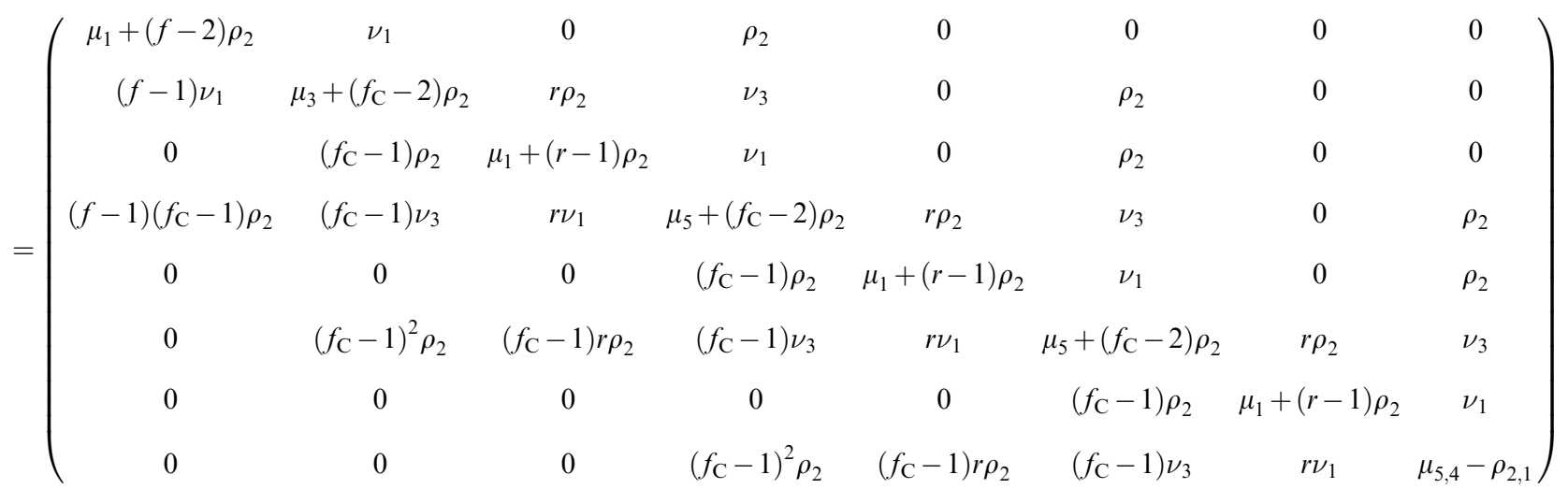

For higher groups the matrices can be constructed iteratively. The matrix $\mathbf{A}_{n}$ is a $2(n-1) \times 2(n-1)$-matrix and it reads

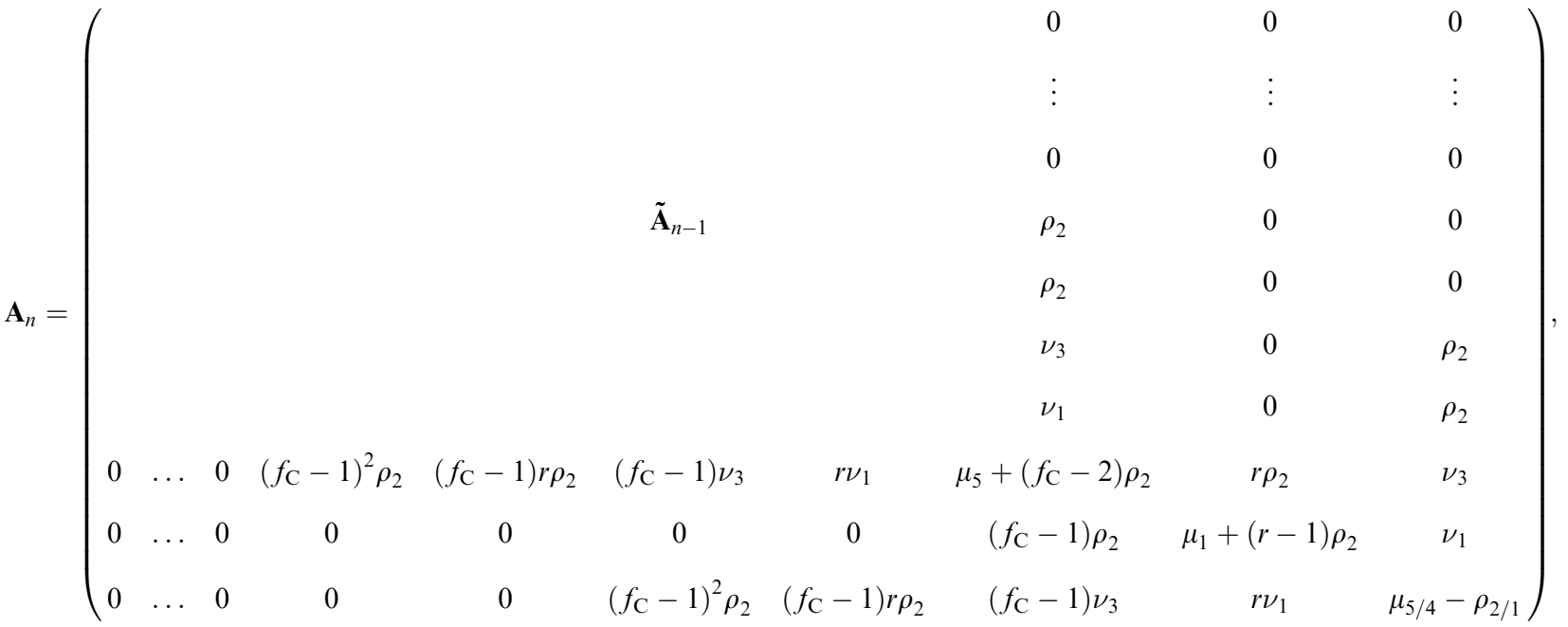

where the matrix $\tilde{\mathbf{A}}_{n-1}$ is obtained by crossing out the last row and the last column of the matrix $\mathbf{A}_{n-1}$. 
The B-matrices of generations four and five read

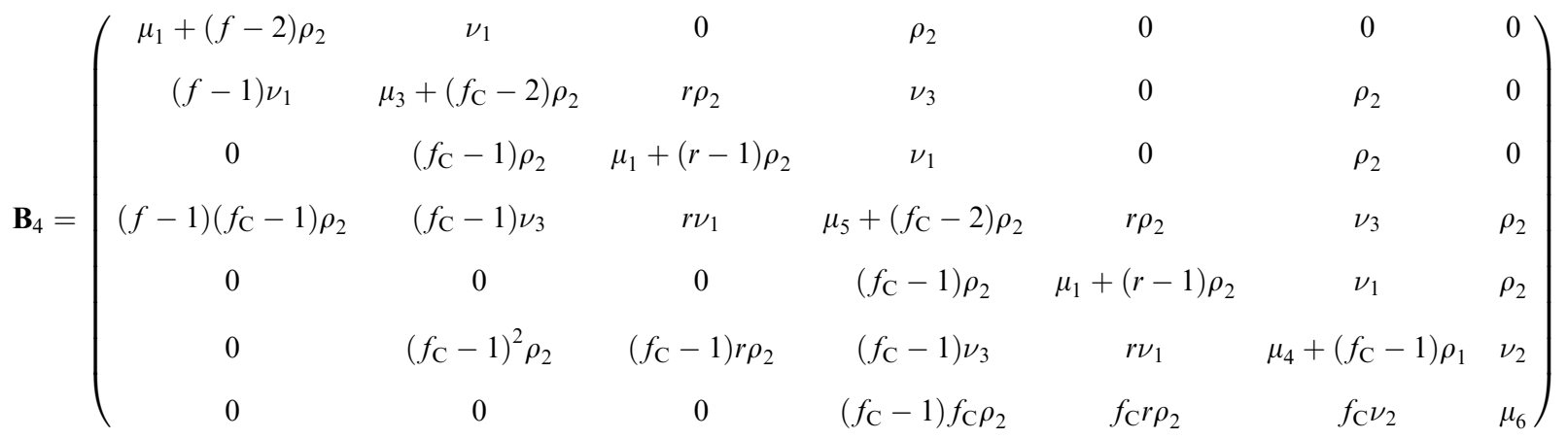

and

$\mathbf{B}_{5}$

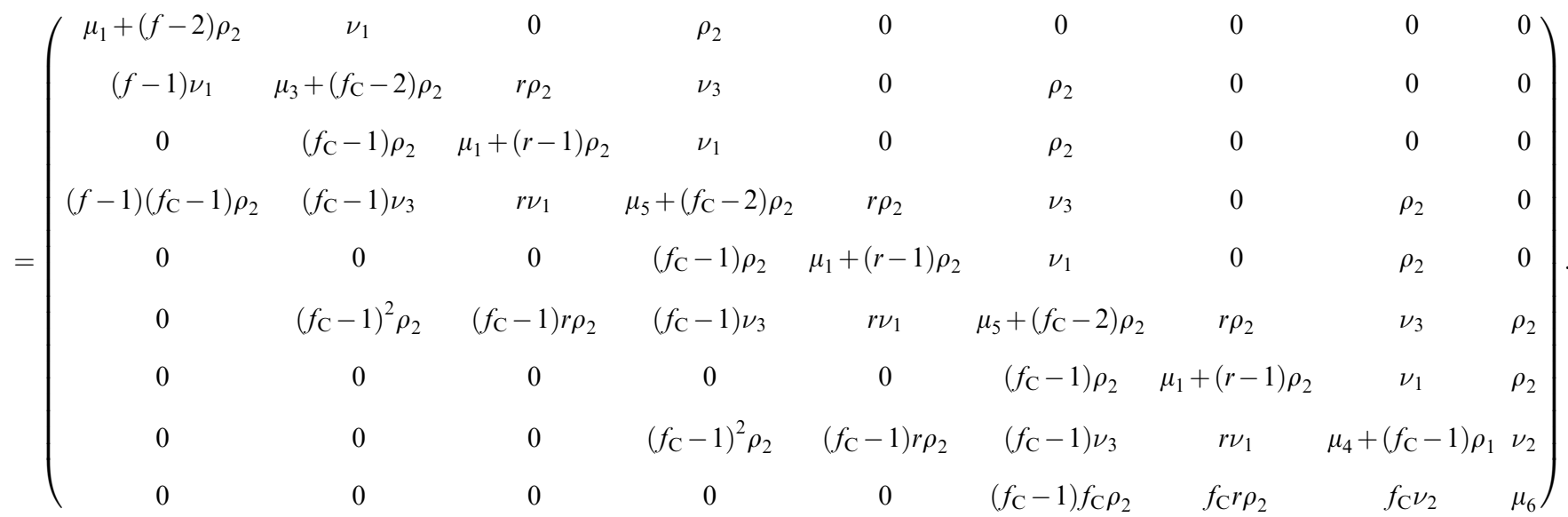

For higher generations, the matrix can be given in an iterative form. The matrix $\mathbf{B}_{n}(n=6, \ldots, G)$ is of the dimension $(2 n-1) \times$ $(2 n-1)$ and reads

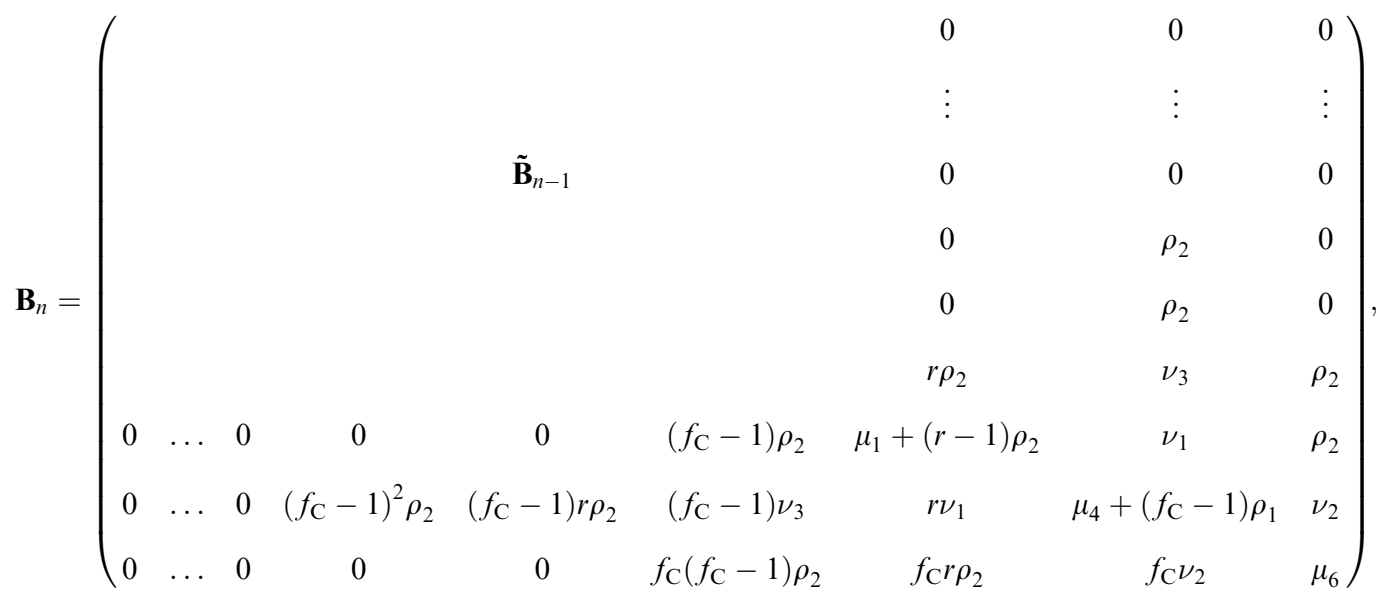

where the matrix $\tilde{\mathbf{B}}_{n-1}$ is obtained by crossing out the last row and the last column of the matrix $\mathbf{B}_{n-1}$ and replacing its last entry (i.e. $\left.\left(\mathbf{B}_{n-1}\right)_{2 n-4,2 n-4}\right)$ by $\mu_{5}+\left(f_{\mathrm{C}}-2\right) \rho_{2}$. 


\section{Acknowledgements}

The authors appreciate the fruitful discussions with Alexander Blumen and Florian Fürstenberg. M. D. acknowledges the support of the Deutsche Forschungsgemeinschaft (Grant No. GRK 1642/1).

\section{References}

1 D. Astruc, E. Boisselier and C. Ornelas, Chem. Rev., 2010, 110, 1857-1959.

2 K. Inoue, Prog. Polym. Sci., 2000, 25, 453-571.

3 F. Vögtle, S. Gestermann, R. Hesse, H. Schwierz and B. Windisch, Prog. Polym. Sci., 2000, 25, 987-1041.

4 E. Buhleier, W. Wehner and F. Vögtle, Synthesis, 1978, 155-158.

5 G. R. Newkome, Z. Yao, G. R. Baker and V. K. Gupta, J. Org. Chem., 1985, 50, 2003-2004.

6 D. A. Tomalia, H. Baker, J. Dewald, M. Hall, G. Kallos, S. Martin, J. Roeck, J. Ryder and P. Smith, Polym. J., 1985, 17, 117-132.

7 C. J. Hawker and J. M. J. Fréchet, J. Am. Chem. Soc., 1990, 112, 7638-7647.

8 M. Sowinska and Z. Urbanczyk-Lipkowska, New J. Chem., 2014, 38, 2147-2708.

9 M. E. Piotti, F. Rivera, R. Bond, C. J. Hawker and J. M. J. Fréchet, J. Am. Chem. Soc., 1999, 121, 9471-9472.

10 S. Hecht, J. Polym. Sci., Part A: Polym. Chem., 2003, 41, 1047-1058.

11 W. R. Dichtel, S. Hecht and J. M. Fréchet, Org. Lett., 2005, 7, 4451-4454.

12 P. Antoni, Y. Hed, A. Nordberg, D. Nyström, H. von Holst, A. Hult and M. Malkoch, Angew. Chem., 2009, 121, 2160-2164.

13 T. Kang, R. J. Amir, A. Khan, K. Ohshimizu, J. N. Hunt, K. Sivanandan, M. I. Montañez, M. Malkoch, M. Ueda and C. J. Hawker, Chem. Commun., 2010, 46, 1556-1558.

14 I. Gadwal and A. Khan, RSC Adv., 2015, 5, 43961-43964.

15 E. Lallana, F. Fernandez-Trillo, A. Sousa-Herves, R. Riguera and E. Fernandez-Megia, Pharm. Res., 2012, 29, 902-921.

16 M. Dolgushev and A. Blumen, J. Chem. Phys., 2009, 131, 044905.

17 R. La Ferla, J. Chem. Phys., 1997, 106, 688-700.

18 A. Kumar and P. Biswas, Macromolecules, 2010, 43, 7378-7385. 19 A. Kumar and P. Biswas, J. Chem. Phys., 2012, 137, 124903. 20 A. Kumar and P. Biswas, Soft Matter, 2013, 9, 2375-2379.

21 G. J. Rai, A. Kumar and P. Biswas, J. Rheol., 2016, 60, 111-120.
22 L. F. Pinto, J. Correa, M. Martin-Pastor, R. Riguera and E. Fernandez-Megia, J. Am. Chem. Soc., 2013, 135, 1972-1977.

23 L. F. Pinto, R. Riguera and E. Fernandez-Megia, J. Am. Chem. Soc., 2013, 135, 11513-11516.

24 F. Mohamed, M. Hofmann, B. Pötzschner, N. Fatkullin and E. A. Rössler, Macromolecules, 2015, 48, 3294-3302.

25 M. Hofmann, C. Gainaru, B. Cetinkaya, R. Valiullin, N. Fatkullin and E. A. Rössler, Macromolecules, 2015, 48, 7521-7534.

26 A. Kumar and P. Biswas, Phys. Chem. Chem. Phys., 2013, 15, 20294-20302.

27 D. A. Markelov, M. Dolgushev, Y. Y. Gotlib and A. Blumen, J. Chem. Phys., 2014, 140, 244904.

28 D. A. Markelov, S. G. Falkovich, I. M. Neelov, M. Y. Ilyash, V. V. Matveev, E. Lähderanta, P. Ingman and A. A. Darinskii, Phys. Chem. Chem. Phys., 2015, 17, 3214-3226.

29 F. Fürstenberg, M. Dolgushev and A. Blumen, J. Chem. Phys., 2012, 136, 154904.

30 C. Cai and Z. Y. Chen, Macromolecules, 1997, 30, 5104-5117.

31 N. L. Biggs, Algebraic graph theory, Cambridge university press, 1993.

32 A. A. Gurtovenko and A. Blumen, Adv. Polym. Sci., 2005, 182, 171.

33 M. Dolgushev and A. Blumen, Macromolecules, 2009, 42, 5378-5387.

34 M. Doi, Introduction to polymer physics, Oxford university press, 1996.

35 A. Perico and M. Guenza, J. Chem. Phys., 1985, 83, 3103-3109.

36 S. H. Glarum, J. Chem. Phys., 1960, 33, 1371-1375.

37 Y. Y. Gotlib and D. A. Markelov, Polym. Sci., Ser. A, 2007, 49, 1137-1154.

38 A. A. Gurtovenko, D. A. Markelov, Y. Y. Gotlib and A. Blumen, J. Chem. Phys., 2003, 119, 7579.

39 Y. Y. Gotlib and D. A. Markelov, Polym. Sci., Ser. A, 2002, 44, 1341-1350.

40 A. A. Gurtovenko, Y. Y. Gotlib and A. Blumen, Macromolecules, 2002, 35, 7481-7491.

41 M. L. Mansfield and W. H. Stockmayer, Macromolecules, 1980, 13, 1713-1715.

42 M. Guenza and A. Perico, Macromolecules, 1992, 25, 5942-5949.

43 M. Dolgushev and A. Blumen, J. Chem. Phys., 2010, 132, 124905.

44 M. Dolgushev, T. Guérin, A. Blumen, O. Bénichou and R. Voituriez, Phys. Rev. Lett., 2015, 115, 208301. 